\title{
Complications associated with implantation and use of totally implantable venous access port in pediatric and adult patients: a scoping review
}

\section{Komplikacije povezane s implantacijom i upotrebom potpuno implantabilnog venskog pristupnog priključka kod dječjih i odraslih pacijenata: sustavni pregled}

\author{
Petra Klanjšek ${ }^{1}$, Katarina Grm², Jasmina Nerat ${ }^{3}$, Leona Cilar ${ }^{4}$ \\ ${ }^{1}$ Fakulteta za zdravstvene vede Univerza v Mariboru, Žitna ulica 15, 2000, Maribor, Slovenija \\ 2 Univerzitetni klinični center, Ljubljanska ulica 5, 2000 Maribor Slovenija \\ ${ }^{3}$ Fakulteta za zdravstvene vede Univerza v Mariboru, Žitna ulica 15, 2000, Maribor, Slovenija \\ ${ }^{4}$ Fakulteta za zdravstvene vede Univerza v Mariboru, Žitna ulica 15, 2000, Maribor, Slovenija
}

\begin{abstract}
Introduction: The use of totally implantable venous access port systems (TIVAPS) is crucial for long-term intravenous treatment in children and adults patients. The purpose of this article is to examine the extent of existing research on complications related to implantation and management of TIVAPS.
\end{abstract}

Methods: A scoping review was performed from September to December in 2019. The MEDLINE, CINAHL and PubMed databases were used to review and analyse the literature using various combinations of keywords and their English-language synonyms. Quantitative clinical research published from 2010 to December 2019 were included in the analysis. The PRISMA-ScR recommendations were followed.

Results: Out of the 219 search results, 26 studies were included in the final analysis. To identify complications associated with the implantation and handling of TIVAPS, free codes were identified by thematic analysis leading to the development of 17 primary grade descriptive subthemes. For the development of the thematic framework, all secondary level sub-topics were synthesized into three main themes: time-bound complications, infection-related complications, and TIVAPS removal complications.

Discussion: Nurses who use TIVAPS on children and adult patients should be aware of the possible complications associated with TIVAPS. Refinement of surgical techniques and improving care process may improve the longevity of the line. Proper and careful management with TIVADs and more frequent observation by nurses are likely to have positive consequences for patients and positive cost implications for the hospital.

Keywords: central venous catheter, nursing, patient safety

Running head: Totally implantable venous access port

\section{Sažetak}

Uvod: Upotreba potpuno ugradivih venskih pristupnih sustava (TIVAPS) iznimno je važna za dugoročno intravensko liječenje djece i odraslih pacijenata. Cilj je ovoga članka ispitati opseg postojećih istraživanja na temu komplikacija povezanih s implantacijom i rukovanjem s potpuno ugradivim venskim pristupnim sustavima.

Metode: Opsežni pregled izvršen je od rujna do prosinca 2019. godine. Baze podataka MEDLINE, CINAHL i PubMed korištene su za pregled i analizu literature uz upotrebu raznih kombinacija ključnih riječi i njihovih sinonima u engleskom jeziku. U analizu uključena su kvantitativna klinička istraživanja objavljena od 2010. do prosinca 2019. godine. Pridržavali smo se preporuka PRISMA-SCR.

Rezultati: Od 219 rezultata pretraživanja, 26 članaka uključeno je u konačnu analizu. Za identifikaciju komplikacija povezanih s implantacijom i rukovanjem potpuno ugradivih venskih pristupnih sustava, tematski smo analizirali otvorene kodove koji su doveli do razvoja 17 opisnih podtema primarnog razreda. Za razvoj tematskog okvira, sve podteme sekundarne razine sintetizirane su u tri glavne teme: vremenski ograničene komplikacije, komplikacije povezane s infekcijom i komplikacije uklanjanja potpuno ugradivih venskih pristupnih sustava.

Rasprava: Medicinske sestre koje upotrebljavaju potpuno ugradiv venski pristupni sustav kod djece i odraslih pacijenata, trebaju biti upoznate s mogućim komplikacijama povezanih s potpuno ugradivim venskim pristupnim sustavima. Usavršavanje kirurških tehnika i postupaka zdravstvene njege mogu poboljšati dugovječnost venskog sustava. Ispravno i pažljivo liječenje potpuno ugradivih venskih pristupnih sustava te češće opažanje imat će pozitivne posljedice za pacijente i implikacije troškova bolnice.

Ključne riječi: središnji venski kateter, njega, sigurnost pacijenata Kratak naslov: Potpuni implantabilni venski pristupni priključak

Autor za korespondenciju/Corresponding author: Leona Cilar, Fakulteta za zdravstvene vede Univerza v Mariboru, Žitna ulica 15, 2000, Maribor, Slovenija, tel.: +3862 $3004759 \cdot$ e-mail: leona. cilar1@um.si

\section{Introduction}

Totally implantable venous access port systems (TIVAPS) are widely used because they provide easy and safe access to the vascular system for various intravenous therapies and nutrition support [1]. The use of these systems is crucial for frequent blood draws and long-term parenteral therapy, such as systemic therapy, parenteral nutrition, administration of blood products, infusions or antibiotics, recurrent intravenous treatment, and other complex tre- 
atments in both adults [2] and pediatric patients [3]. TIVAPS are implanted surgically under general anesthesia or implanted by an interventional radiologist with intravenous sedation [4]. Often, the device is inserted into the vein of the subclavian, meaning that it is located at the front of the chest [5]. TIVAPS are removed when long-term treatment is completed [6].

The TIVAPs are most frequently used in the oncology nursing department, thus oncology nurses must have knowledge and skills needed for managing TIVAPs [7]. In case of healthcare errors, they must report them and follow the established standards and clinical guidelines [8]. The insertion of an atraumatic needle into the TIVAPS, and the injection of the TIVAPS are performed by nurses worldwide. It is important that nurses who administer therapy via TIVAPS are trained in their correct use [2] in both children and adults. Handling TIVAPS is a nursing intervention that requires specific skills and knowledge to avoid adverse events and complications [9]. Nurses need to be aware of many aspects of care, maintenance, and potential complications of TIVAPS to provide quality nursing care. They must fully understand the standard interventions related to TIVAPS, know how to estimate, and prepare a puncture site and recognize the TIVAPS characteristics (position, shape, and size of the chamber) [10].

The use of TIVAPS has given rise to a wide range of complications in many indications. Recognizing and managing complications prevents prolonged hospital stay, improves patient quality of life, and lowers treatment costs [11]. Timely recognition of complications, assessment of the patient's health status, and appropriate handling of the TIVAPS are essential in reducing the likelihood of life-threatening complications [11]. Further, with early recognition, complications can be resolved in a timely manner, thus improving the chances of resolving them without long-term consequences for the patient. This article deals with the complications associated with the implantation and management of TIVAPS. The findings of the study will allow nurses and other healthcare professionals to review evidence-based data on these complications.

\section{Materials and methods}

\section{Aim}

The aim of this article is to examine the extent of existing research on complications related to implantation and management of totally implantable venous access port systems. We designed and developed the following specific research question using the PCC (Population, Concept and Context) mnemonic: Which common complications occur (concept) in pediatric and adult patients (population) during implementation and management of TIVAPS (context)?

\section{Design}

A scoping review was carried out. The findings are reported according to the guidelines of the Preferred Reporting Items for Systematic reviews and Meta-Analyses extension for Scoping Reviews (PRISMA-ScR).

\section{Search methods}

The literature search was conducted in the MEDLINE, ClNAHL and PubMed databases using the following search string (Table 1). The search was carried out on the 20th December 2019. The search strategy was adopted in each database and kept consistent across all searchers.

\begin{tabular}{llccc} 
TABLe [1] & Search strategy & & \\
\hline Number & Key words & MEDLINE & CINAHL & PubMed \\
\hline$\# 1$ & $\begin{array}{l}\text { "subcutaneous } \\
\text { venous port" } \\
\text { OR "totally } \\
\text { implantable } \\
\text { venous access } \\
\text { port" OR } \\
\text { "venous port" } \\
\text { OR "TIVAP*" OR }\end{array}$ & 359 & 124 & 423 \\
& "TIVAD*" & & & \\
\hline \#2 & "infection*" & 735604 & 229560 & 904730 \\
\hline$\# 3$ & "complication" & 967775 & 420125 & 235182 \\
\hline$\# 4$ & \#1 AND \#2 & 121 & 42 & 273 \\
\hline$\# 5$ & \#2 AND \#3 & 141811 & 51893 & 101066 \\
\hline$\# 6$ & \#1 AND \#2 AND \#3 & 85 & 22 & 122 \\
\hline & & & &
\end{tabular}

The inclusion and exclusion criteria are presented in the Table 2.

TABLE [2] Inclusion and exclusion criteria

\begin{tabular}{|c|c|}
\hline \multicolumn{2}{|c|}{ Inclusion criteria } \\
\hline Population & $\begin{array}{l}\text { Adults ( } 19-64 \text { years of age) and } \\
\text { children }(0-18 \text { years of age) with } \\
\text { inserted TIVAP }\end{array}$ \\
\hline Intervention & $\begin{array}{l}\text { Implementation and management of } \\
\text { TIVAPS }\end{array}$ \\
\hline Study type & Quantitative research \\
\hline \multicolumn{2}{|c|}{ Exclusion criteria } \\
\hline \multicolumn{2}{|c|}{$\begin{array}{l}\text { Duplicates, systematic reviews, meta-analyses, qualitative } \\
\text { research }\end{array}$} \\
\hline \multicolumn{2}{|l|}{ Limits } \\
\hline Time limit & $2010-2019$ \\
\hline Text availability & Full text or open access \\
\hline Language & English \\
\hline
\end{tabular}

\section{Search outcomes}

Two reviewers (PK, KG) screened the titles and abstracts using the inclusion criteria. Differences in judgement were resolved through a consensus procedure by two reviewers (PK, KG). All the studies obtained from the bibliographic databases were entered into EndNote X9.3.1, and duplicates were excluded. The full texts of the selected studies that met the inclusion criteria were retrieved for their final 
inclusion by the same researchers (PK, KG) independently. In cases of doubt, the third and fourth reviewers (JN, LC) eli- minated the divergence or recommended reading the full article (Figure 1).

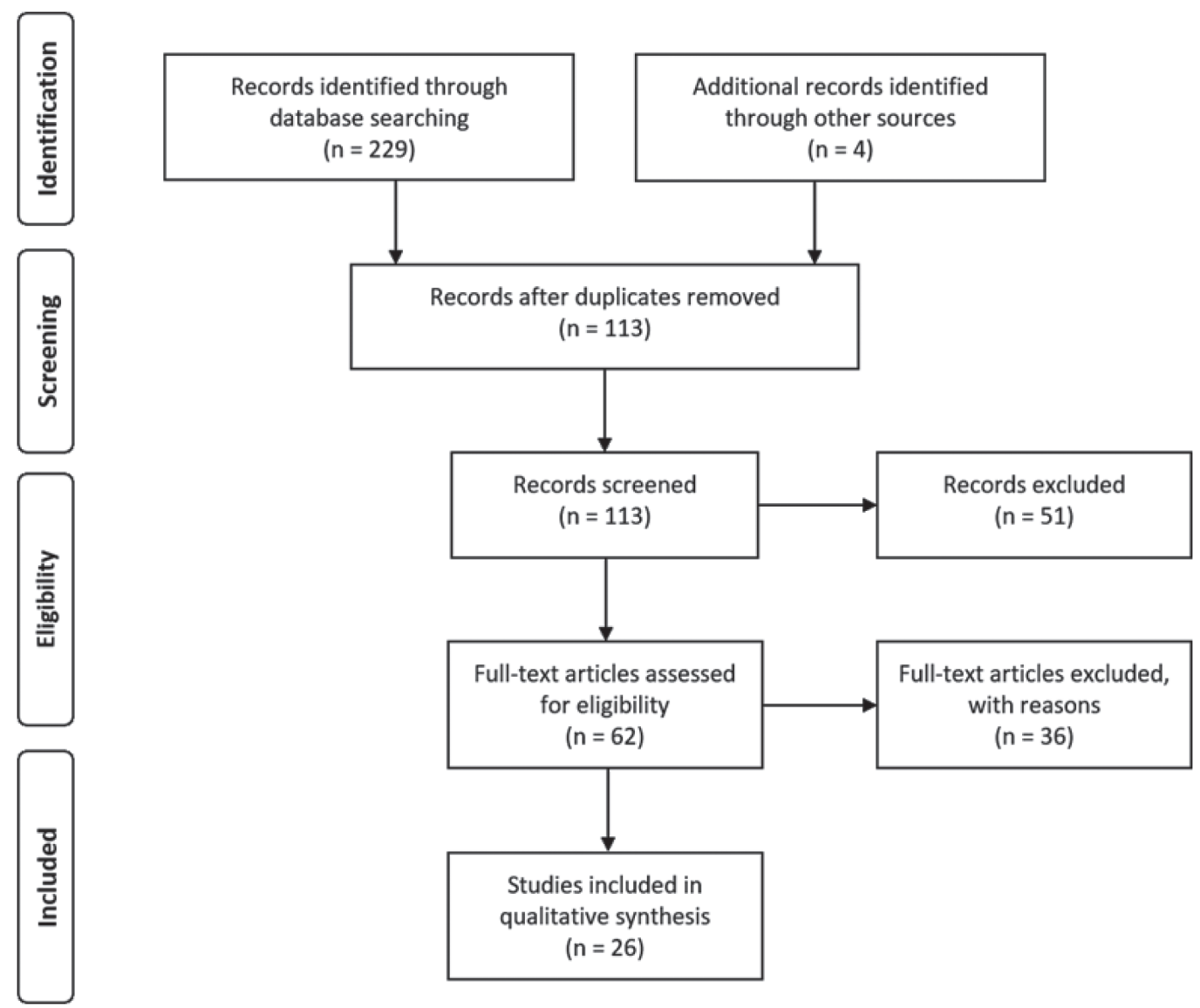

FIGURE 1. PRISMA flow-diagram of the review process

\section{Quality appraisal}

As in other scoping reviews, the aim of this review was not to assess the quality of an individual study, but to explore existing research on complications related to implantation and management of totally implantable venous access port systems. Therefore, the quality appraisal was not performed.

\section{Data abstraction}

The following data was extracted in the form of the table: reference, study design, publication year, country, included population, research design, healthcare workers, and main outcomes.

\section{Synthesis}

Thematic analysis was used for findings synthesis. The data coding process was performed by one reviewer (KG) and checked by the second reviewer (PK). Eventual disagreements were discussed, and consensus was made by the third reviewer (LC). Second, similarities were then identified. Finally, the main themes were developed.

\section{Results}

A total of 26 studies were included in the final literature analysis (Table 3). Research has been conducted in Europe (10), Asia (11) and the United States (2) and published from October 1998 to 2017. The TIVAPS were managed by nurses or qualified healthcare professionals (5), surgeons (6), radiologists (1), surgical and oncology teams (1), and physicians (1). The study with the largest number of patients $(n=1237)$ is the one by Ahn et al. [12], while Granic et al. [21] had the lowest patient count $(n=50)$.

To identify and manage complications associated with the use of TIVAPS, we identified free codes $(n=101)$ and 17 primary grade descriptive subtopics. Primary grade descriptive subtopics were analyzed and grouped into six secondary grade subtopics. All secondary level subtopics were synthesized into three main themes: time-related complications, infection-related complications, and complications related to the removal of the TIVAPS (Table 4). 
TABLE [3] Study characteristics

\begin{tabular}{|c|c|c|c|c|c|c|}
\hline Reference & Study design & $\begin{array}{l}\text { Study time } \\
\text { (Country) }\end{array}$ & $\begin{array}{l}\text { Healthcare } \\
\text { worker }\end{array}$ & $\begin{array}{l}\text { Study population } \\
\text { (number and age) }\end{array}$ & Sample diagnosis & $\begin{array}{l}\text { Sited of insertion of } \\
\text { the TIVAPS }\end{array}$ \\
\hline [12] & $\begin{array}{l}\text { Retrospective } \\
\text { study }\end{array}$ & $\begin{array}{l}\text { August } 2002 \text { - } \\
\text { October } 2009 \text { (Korea) }\end{array}$ & $\begin{array}{l}\text { Interventional } \\
\text { radiologists }\end{array}$ & $\begin{array}{l}n=1237 \\
18-84 \text { years }\end{array}$ & Malignancies & $\begin{array}{l}\text { Right or left internal } \\
\text { jugular vein }\end{array}$ \\
\hline [13] & $\begin{array}{l}\text { Prospective } \\
\text { observational } \\
\text { study }\end{array}$ & $\begin{array}{l}\text { January } \\
2010 \text { - July } 2010 \\
\text { (Japan) }\end{array}$ & Surgeons & $\begin{array}{l}n=59 \\
24-85 \text { years }\end{array}$ & Malignancies & $\begin{array}{l}\text { Subclavian vein, right } \\
\text { or left forearm }\end{array}$ \\
\hline [14] & $\begin{array}{l}\text { Retrospective } \\
\text { study }\end{array}$ & $\begin{array}{l}\text { October } 2000 \\
\text { - January } 2013 \\
\text { (Germany) }\end{array}$ & No data & $\begin{array}{l}n=73 \\
35-86 \text { years }\end{array}$ & Malignancies & $\begin{array}{l}\text { Right or left cephalic } \\
\text { vein, left or right } \\
\text { subclavian vein, left } \\
\text { or right femoralis vein }\end{array}$ \\
\hline [15] & $\begin{array}{l}\text { Retrospective } \\
\text { cohort study }\end{array}$ & $\begin{array}{l}\text { December } 2008 \text { - } \\
\text { July } 2010 \text { (Turkey) }\end{array}$ & No data & $\begin{array}{l}n=347 \\
16-84 \text { years }\end{array}$ & Malignancies & $\begin{array}{l}\text { Subclavian vein, } \\
\text { jugularis vein }\end{array}$ \\
\hline [16] & $\begin{array}{l}\text { Retrospective } \\
\text { study }\end{array}$ & $\begin{array}{l}\text { January } 2001 \text { - } \\
\text { October } 2010 \\
\text { (Greece) }\end{array}$ & $\begin{array}{l}\text { Specially } \\
\text { trained nurses }\end{array}$ & $\begin{array}{l}n=700 \\
17-76 \text { let }\end{array}$ & $\begin{array}{l}\text { Malignancies and } \\
\text { other diseases }\end{array}$ & $\begin{array}{l}\text { Right or left vein of } \\
\text { subclavia, right or left } \\
\text { jugular vein }\end{array}$ \\
\hline [17] & $\begin{array}{l}\text { Retrospective } \\
\text { study }\end{array}$ & $\begin{array}{l}\text { January } 2009 \text { - } \\
\text { March } 2011 \text { (India) }\end{array}$ & No data & $\begin{array}{l}\mathrm{n}=81 \\
/\end{array}$ & No data & $\begin{array}{l}\text { Right or left vein of } \\
\text { subclavia, right or left } \\
\text { jugular vein }\end{array}$ \\
\hline [18] & $\begin{array}{l}\text { Retrospective } \\
\text { cohort study }\end{array}$ & $\begin{array}{l}\text { January - June } 2009 \\
\text { (China) }\end{array}$ & Surgeons & $\begin{array}{l}\mathrm{n}=566 \\
/\end{array}$ & $\begin{array}{l}\text { Malignancies and } \\
\text { haematological } \\
\text { diseases }\end{array}$ & $\begin{array}{l}\text { Cephalic vein, } \\
\text { subclavian vein, } \\
\text { jugular vein and } \\
\text { other veins }\end{array}$ \\
\hline [19] & $\begin{array}{l}\text { Prospective } \\
\text { descriptive } \\
\text { study }\end{array}$ & $\begin{array}{l}\text { February } 2008 \\
\text {-August } 2009 \\
\text { (Italy) }\end{array}$ & Nurses, doctors & $\begin{array}{l}n=1076 \\
18-90 \text { years }\end{array}$ & $\begin{array}{l}\text { Malignancies and } \\
\text { haematological } \\
\text { diseases }\end{array}$ & $\begin{array}{l}\text { Subclavian vein, } \\
\text { jugular vein and } \\
\text { other sites }\end{array}$ \\
\hline [3] & $\begin{array}{l}\text { Retrospective } \\
\text { descriptive } \\
\text { study }\end{array}$ & $\begin{array}{l}2008-2013 \\
\text { (Iran) }\end{array}$ & $\begin{array}{l}\text { Paediatric } \\
\text { surgeon }\end{array}$ & $\begin{array}{l}\mathrm{n}=85 \\
<16 \text { years }\end{array}$ & $\begin{array}{l}\text { Malignancies, } \\
\text { haematological and } \\
\text { other diseases }\end{array}$ & No data \\
\hline [20] & $\begin{array}{l}\text { Retrospective } \\
\text { study }\end{array}$ & $\begin{array}{l}\text { January } 2006 \text { - } \\
\text { October } 2008 \\
\text { (Germany) }\end{array}$ & No data & $\begin{array}{l}n=763 \\
15-87 \text { years }\end{array}$ & $\begin{array}{l}\text { Malignancies and } \\
\text { other diseases }\end{array}$ & No data \\
\hline [21] & $\begin{array}{l}\text { Retrospective } \\
\text { study }\end{array}$ & 2001 - 2012 (Serbia) & $\begin{array}{l}\text { Specially } \\
\text { trained nurses }\end{array}$ & $\begin{array}{l}n=50 \\
28-68 \text { years }\end{array}$ & Malignancies & $\begin{array}{l}\text { Basil vein, cephalic } \\
\text { vein, brachial vein }\end{array}$ \\
\hline [6] & $\begin{array}{l}\text { Retrospective } \\
\text { study }\end{array}$ & $\begin{array}{l}\text { January } 2012 \text { - May } \\
2014 \text { (Turkey) }\end{array}$ & $\begin{array}{l}\text { Cardiovascular } \\
\text { surgeons }\end{array}$ & $\begin{array}{l}\mathrm{n}=347 \\
/\end{array}$ & Malignancies & $\begin{array}{l}\text { Right or left vein } \\
\text { of subclavia, right } \\
\text { jugular vein }\end{array}$ \\
\hline [22] & $\begin{array}{l}\text { Prospective } \\
\text { study }\end{array}$ & $\begin{array}{l}\text { March } 2009 \text { - } \\
\text { December } 2010 \\
\text { (Korea) }\end{array}$ & $\begin{array}{l}\text { Thoracic } \\
\text { surgeon }\end{array}$ & $\begin{array}{l}n=242 \\
14-86 \text { years }\end{array}$ & Malignancies & $\begin{array}{l}\text { The right or left part } \\
\text { of the chest or vein of } \\
\text { the subclavia }\end{array}$ \\
\hline [23] & $\begin{array}{l}\text { Retrospective } \\
\text { study }\end{array}$ & $\begin{array}{l}\text { October } 2004 \text { - } \\
\text { October } 2007 \text { (USA) }\end{array}$ & No data & $\begin{array}{l}n=534 \\
21-95 \text { years }\end{array}$ & $\begin{array}{l}\text { Malignancies and } \\
\text { haematological } \\
\text { diseases }\end{array}$ & Right jugular vein \\
\hline [24] & $\begin{array}{l}\text { Retrospective } \\
\text { study }\end{array}$ & $\begin{array}{l}\text { April } 2006 \text { - June } \\
2009 \\
\text { (Japan) }\end{array}$ & Surgeons & $\begin{array}{l}n=433 \\
/\end{array}$ & Malignancies & Upper arm \\
\hline [25] & $\begin{array}{l}\text { Prospective } \\
\text { study }\end{array}$ & $\begin{array}{l}\text { June } 2004 \text { - January } \\
2014 \text { (UK) }\end{array}$ & $\begin{array}{l}\text { Surgical and } \\
\text { oncology team }\end{array}$ & $\begin{array}{l}n=1000 \\
19-86 \text { years }\end{array}$ & Malignancies & $\begin{array}{l}\text { Left or right } \\
\text { subclavian vein, left } \\
\text { or right jugular vein }\end{array}$ \\
\hline [26] & $\begin{array}{l}\text { Prospective } \\
\text { descriptive } \\
\text { study }\end{array}$ & $\begin{array}{l}\text { Maj } 2006 \text { - April } \\
2007 \\
\text { (France) }\end{array}$ & $\begin{array}{l}\text { Trainee } \\
\text { surgeon and } \\
\text { superior } \\
\text { surgeon }\end{array}$ & $\begin{array}{l}n=815 \\
0,8-85,2 \text { years }\end{array}$ & Malignancies & $\begin{array}{l}\text { Outer jugular vein, } \\
\text { cephalic vein }\end{array}$ \\
\hline [27] & $\begin{array}{l}\text { Retrospective } \\
\text { study }\end{array}$ & 2009 - 2013 (Egypt) & No data & $\begin{array}{l}n=250 \\
16-73 \text { let }\end{array}$ & Malignancies & $\begin{array}{l}\text { Right or left vein of } \\
\text { subclavia, right or left } \\
\text { jugular vein }\end{array}$ \\
\hline
\end{tabular}




\begin{tabular}{|c|c|c|c|c|c|c|}
\hline Reference & Study design & $\begin{array}{l}\text { Study time } \\
\text { (Country) }\end{array}$ & $\begin{array}{l}\text { Healthcare } \\
\text { worker }\end{array}$ & $\begin{array}{l}\text { Study population } \\
\text { (number and age) }\end{array}$ & Sample diagnosis & $\begin{array}{l}\text { Sited of insertion of } \\
\text { the TIVAPS }\end{array}$ \\
\hline [28] & $\begin{array}{l}\text { Retrospective } \\
\text { study }\end{array}$ & $\begin{array}{l}\text { January } 2011 \text { - May } \\
2015 \\
\text { (Slovenia) }\end{array}$ & No data & $\begin{array}{l}\mathrm{n}=193 \\
2 \text { months - 20,7 } \\
\text { years }\end{array}$ & $\begin{array}{l}\text { Malignancies and } \\
\text { haematological } \\
\text { diseases }\end{array}$ & $\begin{array}{l}\text { Right or left } \\
\text { subclavian vein, right } \\
\text { or left outer jugular } \\
\text { vein, right or left } \\
\text { internal jugular vein, } \\
\text { right or left cephalic } \\
\text { vein }\end{array}$ \\
\hline [29] & $\begin{array}{l}\text { Retrospective } \\
\text { study }\end{array}$ & $\begin{array}{l}1998-2014 \\
\text { (Italy) }\end{array}$ & No data & $\begin{array}{l}n=8654 \\
15-98 \text { years }\end{array}$ & $\begin{array}{l}\text { Malignancies and } \\
\text { other diseases }\end{array}$ & Vein subclavia \\
\hline [30] & $\begin{array}{l}\text { Retrospective } \\
\text { study }\end{array}$ & $\begin{array}{l}\text { January } 2013 \text { - July } \\
2015 \\
\text { (Korea) }\end{array}$ & No data & $\begin{array}{l}n=827 \\
18-86 \text { years }\end{array}$ & $\begin{array}{l}\text { Malignancies and } \\
\text { other diseases }\end{array}$ & $\begin{array}{l}\text { Right or left jugular } \\
\text { vein or right or left } \\
\text { vein subclavia }\end{array}$ \\
\hline [31] & $\begin{array}{l}\text { Retrospective } \\
\text { study }\end{array}$ & $\begin{array}{l}\text { November } 2013 \text { - } \\
\text { July } 2016 \\
\text { (China) }\end{array}$ & No data & $\begin{array}{l}\mathrm{n}=270 \\
/\end{array}$ & Malignancies & Vein basilica \\
\hline [32] & $\begin{array}{l}\text { Retrospective } \\
\text { study }\end{array}$ & $\begin{array}{l}\text { March } 2016 \text { - March } \\
2017 \text { (USA) }\end{array}$ & No data & $\begin{array}{l}\mathrm{n}=539 \\
/\end{array}$ & Malignancies & No data \\
\hline
\end{tabular}

TABLE [4] Overview of data synthesis

\begin{tabular}{|c|c|c|c|}
\hline Main topic & $\begin{array}{l}\text { Subtopics of } \\
\text { secondary } \\
\text { degree }\end{array}$ & $\begin{array}{l}\text { Subtopics of primary } \\
\text { degree }\end{array}$ & Free codes \\
\hline \multirow{10}{*}{$\begin{array}{l}\text { Complications } \\
\text { associated with } \\
\text { time }\end{array}$} & \multirow{4}{*}{$\begin{array}{l}\text { Early } \\
\text { complications }\end{array}$} & Wound & Dehiscence, wound problems, or scars. \\
\hline & & Infection & $\begin{array}{l}\text { Bloodstream infection, TIVAPS pocket infection, undetermined } \\
\text { infection, local inflammation. }\end{array}$ \\
\hline & & Mechanical complications & $\begin{array}{l}\text { Catheter displacement, catheter and chamber displacement, } \\
\text { chamber displacement, chamber rotation, catheter closure } \\
\text { due to fibrin layer formation, catheter rupture, mechanical } \\
\text { dysfunction of the chamber, dysfunction of TIVAPS, hematoma, } \\
\text { artery puncture. }\end{array}$ \\
\hline & & Physiological complications & $\begin{array}{l}\text { Thrombosis, pneumothorax, arrhythmia of the heart, bleeding, } \\
\text { cellulitis, phlebitis, pain. }\end{array}$ \\
\hline & \multirow{4}{*}{$\begin{array}{l}\text { Late } \\
\text { complications }\end{array}$} & Wound & $\begin{array}{l}\text { Wound dehiscence, skin necrosis, view of TIVAPS through the } \\
\text { skin, suture disruption. }\end{array}$ \\
\hline & & Infection & $\begin{array}{l}\text { Bloodstream infection (sepsis), TIVAPS pocket infection, } \\
\text { bacteremia, skin infection, undetermined infection. }\end{array}$ \\
\hline & & Mechanical complications & $\begin{array}{l}\text { Catheter displacement, catheter thrombosis, fibrin layer } \\
\text { formation in the catheter, chamber thrombosis, indeterminate } \\
\text { catheter and / or chamber closure, catheter rupture, } \\
\text { extravasation, Pinch-off syndrome, chamber rotation, catheter } \\
\text { de-connection, dysfunction of TIVAPS, irregular puncture in } \\
\text { TIVAPS. }\end{array}$ \\
\hline & & Physiological complications & $\begin{array}{l}\text { Thrombosis, venous thrombosis syndrome, thrombotic } \\
\text { complications, pulmonary thromboembolism, lymphatic } \\
\text { edema, swelling, cellulitis. }\end{array}$ \\
\hline & \multirow[t]{2}{*}{$\begin{array}{l}\text { Indefinite } \\
\text { complications }\end{array}$} & Wound & $\begin{array}{l}\text { Skin erosion, skin diseases, skin dehiscence, view of the TIVAPS } \\
\text { chamber from the wound, necrosis. }\end{array}$ \\
\hline & & Mechanical complications & $\begin{array}{l}\text { Catheter displacement, catheter rupture, chamber rotation } \\
\text { in TIVAPS pocket, hematoma, indeterminate dysfunction } \\
\text { of TIVAPS, TIVAPS closure, catheter closure, systemic arrest, } \\
\text { extravasation, chamber leak, catheter rupture. }\end{array}$ \\
\hline $\begin{array}{l}\text { Infection-related } \\
\text { complications }\end{array}$ & $\begin{array}{l}\text { Complications } \\
\text { of infection }\end{array}$ & Infection & $\begin{array}{l}\text { TIVAPS pocket infection, TIVAPS infection, catheter infection, } \\
\text { bloodstream infection, undetermined infection, undetermined } \\
\text { inflammation, inflammatory syndrome. }\end{array}$ \\
\hline
\end{tabular}




\begin{tabular}{|c|c|c|c|}
\hline Main topic & $\begin{array}{l}\text { Subtopics of } \\
\text { secondary } \\
\text { degree }\end{array}$ & $\begin{array}{l}\text { Subtopics of primary } \\
\text { degree }\end{array}$ & Free codes \\
\hline \multirow{6}{*}{$\begin{array}{l}\text { Complications } \\
\text { related to the } \\
\text { removal of the } \\
\text { TIVAPS }\end{array}$} & \multirow{5}{*}{$\begin{array}{l}\text { Treatment } \\
\text { complications }\end{array}$} & Wound & $\begin{array}{l}\text { Erosion, skin diseases, skin necrosis, view of the chamber } \\
\text { through the skin. }\end{array}$ \\
\hline & & Infection & $\begin{array}{l}\text { Bacteremia, undetermined infection, bloodstream infection, } \\
\text { suspected bloodstream infection. }\end{array}$ \\
\hline & & Treatment & Completion of treatment. \\
\hline & & Mechanical complications & $\begin{array}{l}\text { TIVAPS chamber rotation, TIVAPS dysfunction, chamber and } \\
\text { catheter de-connection, chamber leakage, indeterminate } \\
\text { chamber or catheter movement, catheter termination, } \\
\text { extravasation, unspecified closure, unspecified complications, } \\
\text { catheter rupture. }\end{array}$ \\
\hline & & Physiological complications & $\begin{array}{l}\text { Thrombosis, venous thrombosis syndrome, bleeding, pain, } \\
\text { hematoma, inflammation. }\end{array}$ \\
\hline & $\begin{array}{l}\text { Personal } \\
\text { reasons }\end{array}$ & Psychological causes & Patient will. \\
\hline
\end{tabular}

\section{Discussion}

TIVAPS are widely used systems for chronically ill patients who need long-term treatment. As evident in our findings, TIVAPS are often implemented in patients with chronic illnesses and malignancies. However, complications due to the use of TIVAPS are evident.

After a literature search, analysis and synthesis, the complications associated with the use and handling of TIVAPS were classified as time-related complications (early complications are wound infections, mechanical complications and physiological complications; late complications are wound infections, mechanical complications and physiological complications; indefinite complications are wound and mechanical complications), infection-related complications (complications due to various sites of infections), and complications related to the removal of the TIVAPS (complications due to treatment complications as wound, infection, treatment complications, and physiological or mechanical complications, or personal reasons such as psychological issues).

Complications associated with TIVAPS differ with regards to their time of occurrence. After the insertion and removal of the TIVAPS, permanent scars remain in both children and adult patients [22]. Moreover, early complications associated with TIVAPS insertion are surgical dehiscence and port failure [22]. Serious complicating are infections, thrombosis, obstruction, extravasation, displacement [26], and mechanical dysfunctions such as chamber rotation [12].

Infections due to TIVAPS usage and management are rare; however, they can cause serious life-threatening conditions [3]. Local signs of the infection are evident in only $18 \%$ of patients [33]. Sepsis is suspected if the patient has a fever, hypothermia, tachycardia, tachypnea, or other relevant clinical symptoms [34]. Catheter-related bloodstream infection is a serious complication that increases the risk for patient morbidity and mortality [12], prolongs hospitaliza- tion in an average of 13 days, and increases treatment costs [12]. Parenteral nutrition is more frequently used in patients with TIVAPS related infections [33]. It is evident from the literature analysis that the most frequent infections are TIVAPS pocket infections, TIVAPS infections, catheter infections, bloodstream infections, undetermined infections, undetermined inflammations, and inflammatory syndromes. Early and appropriate treatment can prevent the infection progressing to sepsis and eventual death [34]. Research shows that TIVAPS insertion into vein subclavia and use of shorter length catheters [22]. Lecronier et al. [33] note that TIVAPS infections can often cause preterm removal of TIVAPS, delay of chemotherapy administration or difficulties in specific treatments.

Complications in the removal of TIVAPS can occur in correlation with the treatment or due to personal reasons. Treatment complications are correlated with the wound (erosion, skin diseases, skin necrosis, chamber visible through skin), infection (bacteremia, undetermined infection, bloodstream infection, suspected bloodstream infection), treatment, mechanical complications (TIVAPS chamber rotation, TIVAPS dysfunction, chamber and catheter deconnection, chamber leakage, indeterminate chamber or catheter movement, catheter termination, extravasation, unspecified closure, unspecified complications, catheter rupture) and physiological complications (thrombosis, venous thrombosis syndrome, bleeding, pain, hematoma, inflammation). The risk factors for complications in the process of TIVAPS removal are age (younger people are at higher risk for infection), the time period between the TIVAPS insertion and its first use (there are more complications if the time period is shorter than six days) and the neutropenia-inducing potential of administered chemotherapy regimens (high-risk regimens are correlated with frequent complications) [26]. Removal of the TIVAPS can also be done at the request of the patient $[12,18,6]$. 
In summary, the results of our study provide an overview of the most common complications associated with the implantation and use of TIVAPS. The results are important for nurses who care for patients with inserted TIVAPS. It is their role to identify possible complications for prompt treatment. Pires \& Vasques [7] found out that nurses do not have adequate knowledge of TIVAPS, its purpose, puncture techniques, maintenance, and management. There is also a need for standardizing professional clinical skills. Nurses need to have the appropriate knowledge to be able to educate patients with inserted TIVAPS about proper care for the systems, daily life activities, and signs of complications of the TIVAPS.

\section{Limitations}

The present study has several limitations. Firstly, we did not consider, nor did we analyze non-published papers or those papers which were not freely available. The search string was relatively narrow, so we may have missed relevant papers on the research subject. Moreover, a scoping review does not propose an exhaustive search and the quality assessment of reviewed sources. Thus, the findings of this study should be interpreted with caution. Nevertheless, due to the number of studies included in the final analysis, we believe that this scoping review provides new knowledge and evidence in the field of implementation and management of TIVAPS in pediatric and adults patients.

\section{Conclusion}

TIVAPS offer many benefits in treatment. Properly used, they enable frequent blood draws and safe administration of long-term parenteral therapy, as well as repeated intravenous treatments in both pediatric and adult patients. However, incorrect use of TIVAPS can lead to many complications. It is clear from the literature that fewer complications are associated with infection in TIVAPS implanted into the vein subclavia. Higher stability of the TIVAPS chamber and the catheter is observed with the TIVAPS implanted in the chest, although brachial and arm TIVAPS are now being used more regularly. However, these factors can only be influenced by a doctor or a doctor surgeon, rather than a nurse. The nurse can reduce the occurrence of early, late, and undetermined complications that limit / complicate the use of TIVAPS, as well as complications associated with infections and the removal of TIVAPS, by adhering to standards for the proper handling of TIVAPS performed according to the aseptic method of work in both the clinical setting and on the field.

A nurse administering a therapy via a TIVAPS must be acquainted with the complications that may occur during and after implantation of a TIVAPS, as well as during its use. Nurses are also tasked with providing medical education to patients and their relatives about the complications associated with the implantation and management of TIVAPS. This knowledge will allow the nurse to quickly identify any complications and, consequently, intervene promptly.

\section{Nema sukoba interesa}

\section{Authors declare no conflict of interest}

\section{References}

[1] Tsuruta S., Goto, Y., Miyake, H., Nagai, H., Yoshioka, Y., Yuasa, N., \& Takamizawa, J. Late complications associated with totally implantable venous access port implantation via the internal jugular vein. Supportive Care in Cancer; 2019.

[2] Kao, C. Y., Fu, C. H., Cheng, Y. C., Chen, J. L., Cheng, Y. C., Chen, C. C. C., $\&$ Chai, J. W. Outcome analysis in 270 radiologically guided implantations of totally implantable venous access ports via basilic vein. Journal of the Chinese Medical Association. 2020; 83 (3): 295-301.

[3] Esfahani, H., Ghorbanpor, M., \& Tanasan, A. Implantable port devices, complications and outcome in pediatric cancer, a retrospective study. Iranian journal of pediatric hematology and oncology. 2016; 6 (1): $1-8$.

[4] Walser, E. M. Venous access ports: indications, implantation technique, follow-up, and complications. Cardiovascular and interventional radiology. 2012; 35 (4): 751-764.

[5] Liu, Y., Li, L. L., Xu, L., Feng, D. D., Cao, Y., Mao, X. Y., ... \& Chen, B. Comparison between Arm Port and Chest Port for Optimal Vascular Access Port in Patients with Breast Cancer: A Systematic Review and MetaAnalysis. BioMed Research International. 2020.

[6] Gurkan, S., Seber, S., Gur, O., Yetisyigit, T., Okan Donbaloglu, M., \& Ozkaramanli Gur, D. Retrospective evaluation of totally implantable venous access port devices: early and late complications. J buon. 2015; 20 (1): 338-345.

[7] Pires, N. N., \& Vasques, C. I. Nurses' knowledge regarding the handling of the totally-implanted venous access device. Texto \& Contexto-Enfermagem. 2014; 23 (2): 443-450.
[8] Waller, A., Turon, H., Bryant, J., Shepherd, J., Hobden, B., \& Sanson-Fisher, R. Nurses perspective on healthcare errors in oncology care: A cross-sectional study. European Journal of Oncology Nursing. 2020.

[9] Cardoso, A. F., Moreli, L., Braga, F. T., Vasques, C. I., Santos, C. B., \& Carvalho, E. C. (2012). Effect of a video on developing skills in undergraduate nursing students for the management of totally implantable central venous access ports. Nurse education today. 2012; 32 (6): 709-713.

[10] Blackburn, P. L., \& van Boxtel, T. J. Nursing of vascular access: highlights of hot issues. In Totally Implantable Venous Access Devices (pp. 231-237). Springer, Milano; 2012.

[11] Pinelli, F., Cecero, E., Degl'Innocenti, D., Selmi, V., Giua, R., Villa, G., ... \& Pittiruti, M. Infection of totally implantable venous access devices: a review of the literature. The journal of vascular access. 2018; 19 (3): 230-242.

[12] Ahn, S. J., Kim, H. C., Chung, J. W., An, S. B., Yin, Y. H., Jae, H. J., \& Park, J. H. Ultrasound and fluoroscopy-guided placement of central venous ports via internal jugular vein: retrospective analysis of 1254 port implantations at a single center. Korean journal of radiology. 2012; 13 (3): 314-323.

[13] Akahane, A., Sone, M., Ehara, S., Kato, K., Suzuki, M., Tanaka, R., ... \& Masahiro, K. Central venous port-related infection in patients with malignant tumors: An observational study. Upsala journal of medical sciences. 2012; 117 (3): 300-308.

[14] Almasi-Sperling, V., Hieber, S., Lermann, J., Strahl, O., Beckmann, M. W., Lang, W., \& Sagban, T. A. Femoral placement of totally implanta- 
ble venous access ports in patients with bilateral breast Cancer. Geburtshilfe und Frauenheilkunde. 2016; 76 (1): 53-58.

[15] Aribaş, B. K., Arda, K., Aribaş, Ö., Çiledağ, N., Yoloğlu, Z., Aktaș, E., ... \& Tekin, E. Comparison of subcutaneous central venous port via jugular and subclavian access in 347 patients at a single center. Experimental and therapeutic medicine. 2012; 4(4): 675-680.

[16] Barbetakis, N., Asteriou, C., Kleontas, A., \& Tsilikas, C. Totally implantable central venous access ports. Analysis of 700 cases. Journal of Surgical Oncology. 2011; 104 (6): 654-656.

[17] Bassi, K. K., Giri, A. K., Pattanayak, M., Abraham, S. W., \& Pandey, K. K. Totally implantable venous access ports: retrospective review of long-term complications in 81 patients. Indian Journal of Cancer. 2012; 49 (1): 114-148.

[18] Chang, Y. F., Lo, A. C., Tsai, C. H., Lee, P. Y., Sun, S., Chang, T. H., ... \& Chen, J. R. Higher complication risk of totally implantable venous access port systems in patients with advanced cancer-a single institution retrospective analysis. Palliative medicine. 2013; 27 (2): 185191.

[19] Dal Molin, A., Rasero, L., Guerretta, L., Perfetti, E., \& Clerico, M. The late complications of totally implantable central venous access ports: the results from an Italian multicenter prospective observation study. European Journal of Oncology Nursing. 2011; 15 (5): 377-381.

[20] Goltz, J. P., Scholl, A., Ritter, C. O., Wittenberg, G., Hahn, D., \& Kickuth, R. Peripherally placed totally implantable venous-access port systems of the forearm: clinical experience in 763 consecutive patients. Cardiovascular and interventional radiology. 2010; 33 (6): 11591167.

[21] Granic, M., Zdravkovic, D., Krstajic, S., Kostic, S., Simic, A., Sarac, M., ... \& Kovcin, V. Totally implantable central venous catheters of the port-acath type: complications due to its use in the treatment of cancer patients. J BUON. 2014; 19 (3): 842-846.

[22] Keum, D. Y., Kim, J. B., \& Chae, M. C. Safety of a totally implantable central venous port system with percutaneous subclavian vein access. The Korean journal of thoracic and cardiovascular surgery. 2013; 46 (3): 202.

[23] McNulty, N. J., Perrich, K. D., Silas, A. M., Linville, R. M., \& Forauer, A. R. Implantable subcutaneous venous access devices: is port fixation necessary? A review of 534 cases. Cardiovascular and interventional radiology. 2010; 33 (4): 751-755.

[24] Mori, Y., Nagayama, S., Kawamura, J. I., Hasegawa, S., Tanaka, E., Okabe, $H_{.}, \ldots$ \& Muto, M. A retrospective analysis on the utility and compli- cations of upper arm ports in 433 cases at a single institute. International journal of clinical oncology. 2016; 21 (3): 474-482.

[25] Mudan, S., Giakoustidis, A., Morrison, D., losifidou, S., Raobaikady, R., Neofytou, K., \& Stebbing, J. 1000 Port-A-Cath ${ }^{\circledast}$ placements by subclavian vein approach: single surgeon experience. World journal of surgery. 2015; 39 (2): 328-334.

[26] Narducci, F., Jean-Laurent, M., Boulanger, L., El Bédoui, S., Mallet, Y., Houpeau, J. L. \& Fournier, C. Totally implantable venous access port systems and risk factors for complications: a one-year prospective study in a cancer centre. European Journal of Surgical Oncology (EJSO). 2011; 37 (10): 913-918.

[27] Samad, A. M. A., \& Ibrahim, Y. A. Complications of Port A Cath implantation: a single institution experience. The Egyptian Journal of Radiology and Nuclear Medicine. 2015; 46 (4): 907-911.

[28] Slemenšek, L. Zapleti, povezani z uporabo osrednjega venskega katetra s podkožnim prekatom pri otrocih z rakom: magistrska naloga (Master thesis, Univerza na Primorskem, Fakulteta za vede o zdravju). 2016.

[29] Paprottka, K. J., Voelklein, J., Waggershauser, T., Reiser, M. F., \& Paprottka, P. M. Retrospective outcome analysis of rates and types of complications after 8654 minimally invasive radiological port implantations via the subclavian vein without ultrasound guidance. La radiologia medica. 2019; 124 (9): 926-933.

[30] Kim, D. H., Ryu, D. Y., Jung, H. J., \& Lee, S. S. Evaluation of complications of totally implantable central venous port system insertion. Experimental and therapeutic medicine. 2019; 17 (3): 2013-2018.

[31] Kao, C. Y., Fu, C. H., Cheng, Y. C., Chen, J. L., Cheng, Y. C., Chen, C. C. C., \& Chai, J. W. Outcome analysis in 270 radiologically guided implantations of totally implantable venous access ports via basilic vein. Journal of the Chinese Medical Association. 2020; 83 (3): 295-301.

[32] Skelton IV, W. P., Franke, A. J., Welniak, S., Bosse, R. C., Ayoub, F., Murphy, M., \& Starr, J. S. Investigation of complications following port insertion in a cancer patient population: a retrospective analysis. Clinical Medicine Insights: Oncology, 13. 2019.

[33] Lecronier, M., Valade, S., Bigé, N., de Prost, N., Roux, D., Lebeaux, D. \& Dres, M. Removal of totally implanted venous access ports for suspected infection in the intensive care unit: a multicenter observational study. Annals of intensive care. 2018; 8 (1): 41.

[34] Walsh, K., \& Caple, C.Vascular Access Devices: Assessing to Reduce Risk of Complications. CINAHL Nursing Guide Nursing Practice and Skill. 2015. 